OPEN ACCESS

Edited by:

Peilin Jia,

The University of Texas Health

Science Center at Houston

(UTHealth), United States

Reviewed by:

Yan Yao,

Chinese Academy of Medical

Sciences, China

Jian Zhong Zhou,

The First Affiliated Hospital of Chongqing Medical University,

China

*Correspondence: Changfa Guo

guo.changfa@zs-hospital.sh.cn

Yangyang Zhang

zhangyangyang_wy@vip.sina.com; zhangyangyang_wy@tongji.edu.cn

${ }^{\dagger}$ These authors have contributed equally to this work

Specialty section:

This article was submitted to Bioinformatics and Computational Biology,

a section of the journal Frontiers in Bioengineering and Biotechnology

Received: 19 December 2019 Accepted: 26 February 2020

Published: 12 March 2020

Citation:

Liu B, Shi X, Ding K, Lv M, Qian Y, Zhu S, Guo $C$ and Zhang $Y$ (2020)

The Joint Analysis of Multi-Omics Data Revealed the Methylation-Expression Regulations in Atrial Fibrillation. Front. Bioeng. Biotechnol. 8:187. doi: 10.3389/fbioe.2020.00187

\section{The Joint Analysis of Multi-Omics Data Revealed the Methylation-Expression Regulations in Atrial Fibrillation}

\author{
Ban Liu't, Xin Shi ${ }^{2 \dagger}$, Keke Ding ${ }^{3 \dagger}$, Mengwei Lv4,5, Yongjun Qian', Shijie Zhu', \\ Changfa Guo ${ }^{7 *}$ and Yangyang Zhang ${ }^{5 *}$
}

\begin{abstract}
'Department of Cardiology, Shanghai Tenth People's Hospital, Tongji University School of Medicine, Shanghai, China, ${ }^{2}$ Department of Pediatric Cardiovascular, Xin Hua Hospital, School of Medicine, Shanghai Jiao Tong University, Shanghai, China, ${ }^{3}$ Department of Cardiology, Shanghai Tongji Hospital, Tongji University School of Medicine, Shanghai, China, ${ }^{4}$ Shanghai East Hospital of Clinical Medical College, Nanjing Medical University, Shanghai, China, ${ }^{5}$ Department of Cardiovascular Surgery, Shanghai East Hospital, Tongji University School of Medicine, Shanghai, China, ${ }^{6}$ Department of Cardiovascular Surgery, National Clinical Research Center for Geriatric, West China Hospital, Sichuan University, Chengdu, China, ${ }^{7}$ Department of Cardiovascular Surgery, Zhongshan Hospital, Fudan University, Shanghai, China
\end{abstract}

Atrial fibrillation (AF) is one of the most prevalent heart rhythm disorder. The causes of AF include age, male sex, diabetes, hypertension, valve disease, and systolic/diastolic dysfunction. But on molecular level, its mechanisms are largely unknown. In this study, we collected 10 patients with persistent atrial fibrillation, 10 patients with paroxymal atrial fibrillation and 10 healthy individuals and did Methylation EPICBead Chip and RNA sequencing. By analyzing the methylation and gene expression data using machine learning based feature selection method Boruta, we identified the key genes that were strongly associated with $\mathrm{AF}$ and found their interconnections. The results suggested that the methylation of KIF15 may regulate the expression of PSMC3, TINAG, and NUDT6. The identified AF associated methylation-expression regulations may help understand the molecular mechanisms of AF from a multi-omics perspective.

Keywords: atrial fibrillation, methylation, multi-omics, feature selection, classification

\section{INTRODUCTION}

Atrial fibrillation (AF), one of the most prevalent heart rhythm disorders, is a potential cause of heart failure and ischemic stroke with high morbidity and mortality (Ogawa et al., 2017; Asmarats et al., 2019). The cause of AF is multifactorial which include age, male sex, diabetes, hypertension, valve disease, and systolic/diastolic dysfunction (Schnabel et al., 2009; Lip et al., 2013; Voukalis et al., 2016). Depends on how often atrial fibrillation occurs and how it responds to treatment, AF is roughly divided into two major subtypes-paroxysmal atrial fibrillation (PAF) and persistent atrial fibrillation (PeAF). In the treatment of $\mathrm{AF}$, drugs were the first choice, non-drug therapies were used only when drug therapy failed or patients could not tolerate the medication. In contrast to the extensive knowledge of etiology, the underlying mechanism of AF remains elusive. Further study of the potential mechanisms of AF could provide novel strategies for the treatment and management to increase quality of life and reduce economic burden of social (Chugh et al., 2001). 
With the development of next generation sequencing (NGS) technologies, growing evidence have demonstrated that $\mathrm{AF}$ is a disease with a significant genetic contribution. Previous studies have filtered novel genetic variants and candidate genes including transcriptional factor genes (PITX2, PRRX1, ZFHX3, NKX2.5, TBX5), ion channel genes (KCNN3, HCN4, CACNA1C, SCN5A, $K C N Q 1, K C N H 2)$, and caveolin genes (CAV1 and CAV2) (Ellinor et al., 2010; Olesen et al., 2014; Sinner et al., 2014; Ma et al., 2016; Nielsen et al., 2018). However, these genes explain only a small fraction of the biology and genetic underpinnings of AF.

Epidemiological studies have demonstrated that genetic, environmental, behavioral, and clinical factors contribute to AF pathogenic mechanism (Zhong et al., 2016). Emelia J. $B$ performed genome-wide methylation using whole blood samples from 183 prevalent AF and 220 incident AF cases. They examined the association between DNA methylation and GWAS loci, suggesting DNA methylation might be a possible mechanism through which AF-specific genetic variations affect gene regulation (Lin et al., 2017). To date, only a few studies have investigated differential DNA methylation as a predictor biomarker at specific candidate loci that were previously associated with AF.

Therefore, we applied DNA methylation profiling study to identify the likely rare damaging variants and putative candidate genes from 10 patients with persistent atrial fibrillation (PeAF), 10 patients with paroxymal atrial fibrillation (PAF) and 10 healthy individuals. Interestingly, we identified top 10 genes (KIF15, ABCA3, FOXG1, VGF, PDE4D, EIF3C, CNTNAP5, SHOX2, VGF, TRIM59) as functional candidate genes and the expression level are significantly increased in PeAF and PAF patients than control. Given the importance of DNA methylation to gene expression, we investigated the gene expression of the same participants using RNA sequencing. We also defined top 10 genes (EPN3, EMD, SMCO4, F2RL2, TMED1, PSMC3, PDZD11, NUDT6, TINAG, GALNT5) in gene expression data and the expression pattern of these genes was significantly different between PeAF and PAF. These results have improved our understanding of the underlying mechanism and offer new insights into the potential pathway of $\mathrm{AF}$, which could provide novel therapeutic option for this disease.

\section{MATERIALS AND METHODS}

\section{Atrial Fibrillation Patients}

Ten patients with paroxymal atrial fibrillation (g1), 10 patients with persistent atrial fibrillation (continuous atrial fibrillation lasting more than 12 months) (g2) and 10 healthy individuals (g3) were enrolled in this study (Table 1). All patients were subjected to detailed medical evaluation, which included medical history, physical examination, electrocardiography (ECG), and echocardiography. Patients with chronic heart failure, coronary heart disease, cardiomyopathy, hyperthyroidism or chronic pulmonary heart disease were excluded.

The study was conducted in accordance with the Declaration of Helsinki, and the protocol used to collect human heart tissue was approved by the Ethics Committee of Shanghai East Hospital (DI: 0402017).

Written informed consents to participate in this study were provided by all the enrolled patients before operation of fibrillation ablation. The left atrial appendage tissues which were abandoned during isolated surgical ablation were collected. Normal left atrial appendages were collected from healthy male donors.

\section{The Methylation Profiles}

The DNA methylation status of $850 \mathrm{~K}$ probes in the 30 samples was measured using Methylation EPICBead Chip. The raw data was quality controlled and preprocessed using R/Bioconductor package minfi $^{1}$ (Aryee et al., 2014). The beta value ranged from 0 to 1 was calculated to represent how each position was methylated. 1 meant high methylation and 0 meant low methylation.

\section{The RNA Sequencing Profiles}

The total RNAs were extracted using RNeasy Mini Kit (Cat\#74106, Qiagen) and the RNA integrity was checked using Agilent Bioanalyzer 2100 (Agilent technologies, Santa Clara, CA, United States). Qualified total RNA was further purified by RNAClean XP Kit (Cat A63987, Beckman Coulter Inc., Kraemer Boulevard, Brea, CA, United States) and RNase-Free DNase Set (Cat\#79254, QIAGEN, GmBH, Germany). Pair-end sequencing reads were generated using Illumina data collection software. First, the reads were mapped onto human reference genome GRCh38 using Hisat2 (version:2.0.4²) (Kim et al., 2015). Then, Stringtie (version:1.3.0 ${ }^{3}$ ) (Pertea et al., 2015) was used to calculate the FPKM (Fragments Per Kilobase of exon model per Million mapped reads).

\section{Feature Selection Algorithm}

There were 866,091 methylation probes and 50,868 RNAs. The number of features were extremely large. It was difficult to select key features using traditional statistical methods. Therefore, we adopted the latest machine learning based feature selection method Boruta to get the key methylation probes and RNAs.

Boruta (Kursa and Rudnicki, 2010) is a feature selection method based on random forest. It can select sample group relevant features effectively. First, it will shuffle the features to create many permuted datasets. Then, it will evaluate the importance score of each feature in the original actual dataset and permuted datasets. Then, it will compare the actual importance score with permuted scores and find the features with significantly higher actual importance scores than permuted scores. After multiple iterations, it will select all the sample group relevant features. The python code from https:/github. com/scikit-learn-contrib/boruta_py was used to apply the Boruta feature selection algorithm.

\footnotetext{
${ }^{1}$ https://bioconductor.org/packages/minfi/

${ }^{2}$ http://ccb.jhu.edu/software/hisat2/

${ }^{3}$ http://ccb.jhu.edu/software/stringtie/
} 
TABLE 1 | Demographic characteristics of AF patients.

\begin{tabular}{|c|c|c|c|c|c|c|c|c|c|c|c|}
\hline No. & $\begin{array}{c}\text { Age } \\
\text { (years) }\end{array}$ & Gender & $\begin{array}{c}\text { Weight } \\
\text { (Kg) }\end{array}$ & $\begin{array}{l}\text { Height } \\
\text { (cm) }\end{array}$ & Smoking & Hypertension & $\begin{array}{l}\text { Diabetes } \\
\text { mellitus }\end{array}$ & $\begin{array}{c}\text { Coronary } \\
\text { angiography } \\
\text { or CTA }\end{array}$ & $\begin{array}{l}\text { LVEF } \\
(\%)\end{array}$ & $\begin{array}{c}\text { Left atrial } \\
\text { diameter }(\mathrm{mm})\end{array}$ & $\begin{array}{l}\text { Duration of } \\
\text { AF (years) }\end{array}$ \\
\hline 1 & 69 & Male & 76 & 169 & No & Yes & No & Negative & 70 & 40 & - \\
\hline 2 & 63 & Male & 64 & 170 & No & No & No & Negative & 59 & 46 & - \\
\hline 3 & 63 & Male & 70 & 170 & No & No & No & Negative & 66 & 39 & - \\
\hline 4 & 69 & Male & 67 & 173 & No & Yes & No & Negative & 67 & 46 & - \\
\hline 5 & 69 & Male & 75 & 165 & No & No & No & Negative & 70 & 36 & - \\
\hline 6 & 61 & Male & 76 & 176 & No & Yes & Yes & Negative & 60 & 42 & - \\
\hline 7 & 64 & Male & 52 & 168 & Yes & No & No & Negative & 64 & 40 & - \\
\hline 8 & 64 & Male & 71 & 181 & No & Yes & Yes & Negative & 63 & 39 & - \\
\hline 9 & 61 & Male & 87 & 167 & Yes & Yes & No & Negative & 62 & 37 & - \\
\hline 10 & 66 & Male & 82 & 173 & No & Yes & No & Negative & 63 & 42 & - \\
\hline 11 & 63 & Male & 86 & 176 & No & Yes & No & Negative & 57 & 46 & 2.5 \\
\hline 12 & 63 & Male & 80 & 178 & No & No & No & Negative & 68 & 55 & 3 \\
\hline 13 & 64 & Male & 70 & 170 & No & No & No & Negative & 67 & 41 & 4 \\
\hline 14 & 64 & Male & 84 & 164 & No & Yes & No & Negative & 55 & 48 & 2 \\
\hline 15 & 65 & Male & 73 & 169 & No & Yes & No & Negative & 69 & 55 & 3.5 \\
\hline 16 & 66 & Male & 66 & 168 & No & Yes & No & Negative & 64 & 45 & 4 \\
\hline 17 & 67 & Male & 80 & 175 & No & Yes & Yes & Negative & 59 & 47 & 2.5 \\
\hline 18 & 67 & Male & 73 & 165 & Yes & Yes & No & Negative & 59 & 47 & 3 \\
\hline 19 & 63 & Male & 61 & 164 & No & No & No & Negative & 73 & 49 & 2 \\
\hline 20 & 67 & Male & 90 & 178 & No & Yes & No & Negative & 70 & 58 & 2.5 \\
\hline
\end{tabular}

LVEF, left ventricular ejection fraction; AF, atrial fibrillation; CTA, CT angiography.

\section{Classification Predictor}

To evaluate how well the selected features can classify the samples, we built an SVM (Support Vector Machine) classifier using the methylation data and another RNA-Seq data based SVM classifier. The svm function in R package e $10171^{4}$ (Chang and Lin, 2011) was used to apply the SVM classification algorithm.

LOOCV (leave-one-out cross validation) was used to objectively evaluate the classification performance. Each time, one sample was treated as test sample while all the other samples were used to train the model. After 30 rounds, all samples had been tested once and the overall accuracy was calculated based on the confusion matrix. In confusion matrix, the actual sample groups were compared with predicted sample groups.

\section{RESULTS}

\section{The Key Methylation Features Identified With Boruta}

We ran Boruta feature selection algorithm on the methylation data and got 10 key methylation features. These 10 key methylation features were listed in Table 2. The probes were annotated to genome positions (Genome Build 37) and genes using the official annotation file from Illumina. Sometime, one position may be associated with multiple genes. Therefore, the 10 methylation probes can be mapped onto 15 gene symbols.

${ }^{4}$ https://CRAN.R-project.org/package $=$ e1071
We checked the GO annotation of these genes and found that cg16703882 (SHOX2) was associated with GO:0007507: heart development which was closely relevant to AF.

We plotted the heatmap of these 10 key methylation features in Figure 1. It can be seen that most of the 10 patients with paroxymal atrial fibrillation (g1), 10 patients with persistent atrial fibrillation (g2) and 10 healthy individuals (g3) were cluster into the right groups.

\section{The Key Gene Expression Features Identified With Boruta}

Similarly, we ran Boruta feature selection algorithm on the RNASeq gene expression data and got 10 key gene expression features.

TABLE 2 | The 10 key methylation features identified by Boruta.

\begin{tabular}{lcccl}
\hline ILMNID & Chromosome & Position & Strand & UCSC Ref gene name \\
\hline cg00702638 & 3 & 44803293 & R & KIF15; KIAA1143 \\
cg02331561 & 16 & 2391081 & F & ABCA17P; ABCA3 \\
cg02991338 & 14 & 29236017 & R & FOXG1 \\
cg04084157 & 7 & 100809049 & F & VGF \\
cg05995159 & 5 & 59325256 & R & PDE4D \\
cg06357615 & 16 & 28403195 & R & MIR6862-2; MIR6862-1; \\
& & & & EIF3CL; EIF3C \\
cg11344566 & 2 & 124782885 & F & CNTNAP5 \\
cg16703882 & 3 & 157823479 & R & SHOX2 \\
cg21186299 & 7 & 100808810 & R & VGF \\
cg26856080 & 3 & 160167746 & R & TRIM59
\end{tabular}




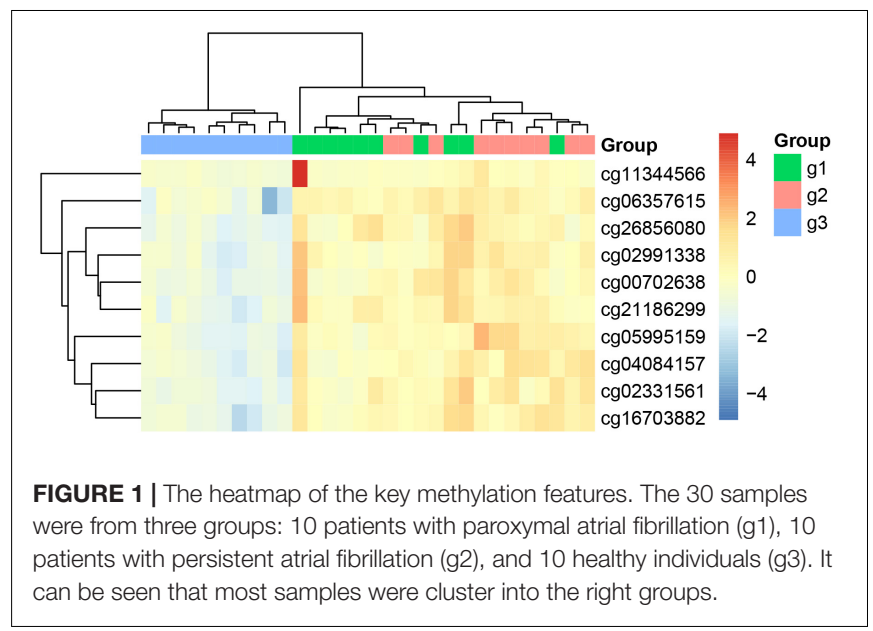

The 10 key gene expression features were given in Table 3. We also plotted their heatmap in Figure 2. The clusters were also largely correct.

\section{The Classification Performance of Methylation and Gene Expression}

From Figures 1, 2, we can see that both methylation and gene expression features can correctly cluster most samples. But we would like to evaluate their performance objectively and quantitatively. Therefore, we applied LOOCV to test the SVM classifiers of methylation and gene expression features. The confusion matrixes of methylation features and gene expression features were listed in Tables 4, 5, respectively.

From Table 3, we can see that the AF patients $(g 1+g 2)$ and healthy individuals (g3) were perfectly classified using the methylation features, but the methylation data did not have great performance on classifying the subtype of AF (g1 and g2). From Table 4, we can see that the two subtype of AF, paroxysmal atrial fibrillation (g1) and persistent atrial fibrillation (g2), had very different gene expression pattern. In other words, the methylation data and gene expression data complement each other. The methylation data can be used to predict the AF and the gene expression data can be used to classify the subtypes of AF.

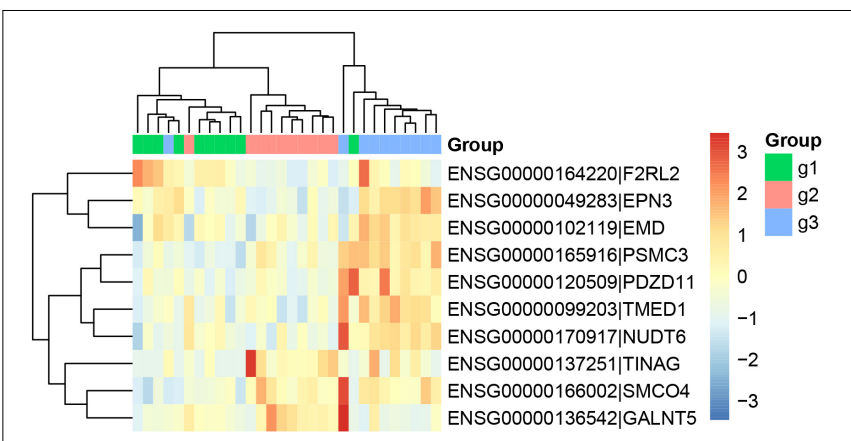

FIGURE 2 | The heatmap of the key gene expression features. The 30 samples were from three groups: 10 patients with paroxymal atrial fibrillation (g1), 10 patients with persistent atrial fibrillation (g2), and 10 healthy individuals (g3). It can be seen that most samples were cluster into the right groups.

TABLE 4 | The confusion matrix of key methylation features.

\begin{tabular}{lccc}
\hline & Predicted g1 & Predicted g2 & Predicted g3 \\
\hline Actual g1 & 8 & 2 & 0 \\
Actual g2 & 3 & 7 & 0 \\
Actual g3 & 0 & 0 & 10 \\
\hline
\end{tabular}

TABLE 5 | The confusion matrix of key gene expression features.

\begin{tabular}{lccc}
\hline & Predicted g1 & Predicted g2 & Predicted g3 \\
\hline Actual g1 & 9 & 0 & 1 \\
Actual g2 & 0 & 9 & 1 \\
Actual g3 & 1 & 0 & 9 \\
\hline
\end{tabular}

We checked the wrongly predicted samples in Tables 3, 4 . They were different. Within the 30 samples, 22 samples had the same predicted labels by expression and methylation. All these 22 samples were correctly predicted. For the 8 inconsistent samples between expression and methylation predictions, at least one of the two predictions (the expression-based prediction and the methylation-based prediction) was correct. In other words, all the samples can be corrected classified based on either expression or methylation. The expression-based prediction and the methylation-based prediction were complementary.

TABLE 3 | The 10 key gene expression features identified by Boruta.

\begin{tabular}{|c|c|c|c|}
\hline Gene ID & Name & Description & GRCh38 locus \\
\hline ENSG00000049283 & EPN3 & Epsin 3 & $17: 50532543-50543750$ \\
\hline ENSG00000102119 & EMD & Emerin & $X: 154379197-154381523$ \\
\hline ENSG00000166002 & SMCO4 & Single-pass membrane protein with coiled-coil domains 4 & $11: 93478472-93543508$ \\
\hline ENSG00000164220 & F2RL2 & Coagulation factor II (thrombin) receptor-like 2 & $5: 76615482-76623434$ \\
\hline ENSG00000099203 & TMED1 & Transmembrane p24 trafficking protein 1 & 19:10832438-10836318 \\
\hline ENSG00000165916 & PSMC3 & Proteasome 265 subunit, atpase 3 & $11: 47418769-47426473$ \\
\hline ENSG00000120509 & PDZD11 & PDZ domain containing 11 & X:70286595-70290514 \\
\hline ENSG00000170917 & NUDT6 & Nudix hydrolase 6 & $4: 122888697-122922968$ \\
\hline ENSG00000137251 & TINAG & Tubulointerstitial nephritis antigen & 6:54307859-54390152 \\
\hline ENSG00000136542 & GALNT5 & Polypeptide N-acetylgalactosaminyltransferase 5 & $2: 157257598-157314211$ \\
\hline
\end{tabular}




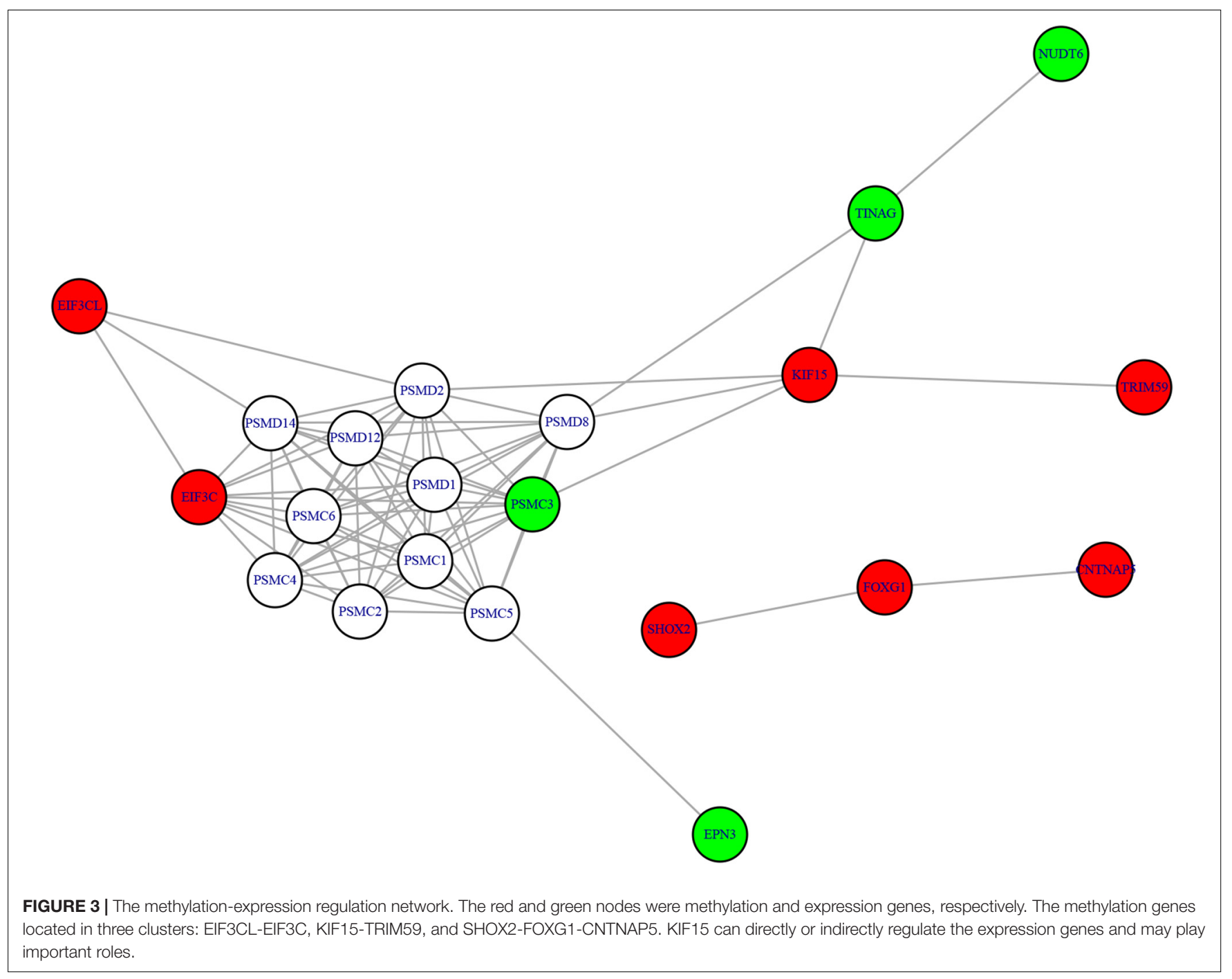

\section{The Methylation-Expression Regulation Network}

We mapped the genes of methylation features and gene expression features to the STRING network (Version 11.0 ${ }^{5}$ ) (Szklarczyk et al., 2018) and visualized the network using R package igraph (Csardi and Nepusz, 2006) ${ }^{6}$ to identify the potential relationship between two candidate genes sets. The methylation-expression regulation network was shown in Figure 3. In the network, the methylation and expression genes were marked in red and green. The methylation genes located in three clusters: EIF3CL-EIF3C, KIF15-TRIM59, and SHOX2-FOXG1-CNTNAP5. The three expression genes (PSMC3, TINAG, and NUDT6) were connected with methylation gene KIF15. Even EPN3 can be indirectly connected to KIF15. That made KIF15 at the center of the network. These results suggested that KIF5 may play important roles in the pathogenesis of AF.

\footnotetext{
${ }^{5} \mathrm{http}: / /$ string-db.org

${ }^{6}$ https://CRAN.R-project.org/package=igraph
}

\section{DISCUSSION}

DNA methylation, a pre-transcriptional modification characterized by the addition of methyl groups to specific nucleotides, regulates the stability of gene expression states and maintains genome integrity by collaborating with proteins that modify nucleosomes (Ma et al., 2014; Tao et al., 2016; Shen et al., 2017). Previous studies considered that changes in DNA methylation states contribute to the regulation of biological processes underlying AF, such as fibrosis, atrial dilatation, atrial fibroblast proliferation and differentiation from fibroblasts into myofibroblasts (Zhao et al., 2017). To further enhance the biological understanding of the atrial fibrillation, our study focused on DNA methylation, particularly with respect to how it relates to mRNA expression. Among our two gene sets of top 10 genes, we found PDED4, SHOX2, and EMD were the most important genes for $\mathrm{AF}$ which have been reported associated with AF in previous reference.

Atrial fibrillation is reported to be associated with a profound remodeling of membrane receptors and alterations in cAMP 
dependent regulation of $\mathrm{Ca} 2^{+}$handling. PDE4 is expressed in human atrial myocytes and accounts for approximately $15 \%$ of PDE (phosphodiesterase) activity (Molina et al., 2012). PDE4D encoded protein has 3',5'-cyclic-AMP phosphodiesterase activity and degrades cAMP, which acts as a signal transduction molecule in multiple cell types and represents the major PDE4 subtype (Berk et al., 2016). The activity of PDEs decreased with age, and the relative PDED4 activity was lower in patients with permanent atrial fibrillation than in age-matched sinus rhythm controls (Milton et al., 2011). Previous study provided evidence that patients with pAF were found to have a decreased PDE4 activity as compared with patients in sinus rhythm (Yeh et al., 2007).

Short Stature Homeobox 2 (SHOX2) is a member of the homeobox family of genes in which mutations associated with early-onset and familial AF (Hoffmann et al., 2019). SHOX2 is considered as a key regulator of sinus node development of which deficiency could lead to bradycardia in animal models (VicenteSteijn et al., 2017). Previous study demonstrated SHOX2 was susceptible for SND and AF by screening 98 SND patients and 450 individuals with AF. In the heart development of mouse and zebrafish, they also proved SHOX2 plays an important role, the mutation of SHOX2 could lead to severe bradycardia (Blaschke et al., 2007; Ye et al., 2015).

Emerin (EMD) encodes a serine-rich nuclear membrane protein which located on the cytoplasmic surface of the inner nuclear membrane and related to X-linked Emery-Dreifuss muscular dystrophy (EDMD) (Capanni et al., 2009). Previous study found a nonsense mutation in EMD from two EDMD families which is associated with $\mathrm{X}$-linked recessive inheritance, result in serious cardiac complication, including AF (Sakata et al., 2005). Cardiologic assessment revealed slow atrial fibrillation in a recent case of a 65 -year-old male patient with a hemizygous duplication of 5 bases in exon 6 of the EMD, gene on

\section{REFERENCES}

Aryee, M. J., Jaffe, A. E., Corrada-Bravo, H., Ladd-Acosta, C., Feinberg, A. P., Hansen, K. D., et al. (2014). Minfi: a flexible and comprehensive bioconductor package for the analysis of infinium DNA methylation microarrays. Bioinformatics 30, 1363-1369. doi: 10.1093/bioinformatics/btu049

Asmarats, L., Cruz-Gonzalez, I., Nombela-Franco, L., Arzamendi, D., Peral, V., Nietlispach, F., et al. (2019). Recurrence of device-related thrombus after percutaneous left atrial appendage closure. Circulation 140, 1441-1443. doi: 10.1161/CIRCULATIONAHA.119.040860

Berk, E., Christ, T., Schwarz, S., Ravens, U., Knaut, M., and Kaumann, A. J. (2016). In permanent atrial fibrillation, PDE3 reduces force responses to 5HT, but PDE3 and PDE4 do not cause the blunting of atrial arrhythmias. $\mathrm{Br}$. J. Pharmacol. 173, 2478-2489. doi: 10.1111/bph.13525

Blaschke, R. J., Hahurij, N. D., Kuijper, S., Just, S., Wisse, L. J., Deissler, K., et al. (2007). Targeted mutation reveals essential functions of the homeodomain transcription factor Shox2 in sinoatrial and pacemaking development. Circulation 115, 1830-1838. doi: 10.1161/CIRCULATIONAHA.106.637819

Brisset, M., Ben Yaou, R., Carlier, R. Y., Chanut, A., Nicolas, G., Romero, N. B., et al. (2019). X-linked Emery-Dreifuss muscular dystrophy manifesting with adult onset axial weakness, camptocormia, and minimal joint contractures. Neuromuscul. Disord. 29, 678-683. doi: 10.1016/j.nmd.2019.06.009

Capanni, C., Del Coco, R., Mattioli, E., Camozzi, D., Columbaro, M., Schena, E., et al. (2009). Emerin-prelamin a interplay in human fibroblasts. Biol. Cell 101, 541-554. doi: 10.1042/BC20080175 the X chromosome (Kissel et al., 2009; Zhao et al., 2014; Brisset et al., 2019).

\section{DATA AVAILABILITY STATEMENT}

The raw data supporting the conclusions of this article will be made available by the authors, without undue reservation, to any qualified researcher.

\section{ETHICS STATEMENT}

The studies involving human participants were reviewed and approved by the Ethics Committee of Shanghai East Hospital. The patients/participants provided their written informed consent to participate in this study. Written informed consent was obtained from the individual(s) for the publication of any potentially identifiable images or data included in this manuscript.

\section{AUTHOR CONTRIBUTIONS}

YZ and CG conceived and designed the experiments. BL, XS, and KD performed the experiments. ML, YQ, and SZ analyzed the data. BL, XS, and YZ wrote the manuscript. All authors read and approved the final version of the manuscript.

\section{FUNDING}

The present study was funded by the National Key Research and Development Program (Grant No. 2018-YFC-1312505 to YZ), the National Natural Science Foundation of China (Grant No. 81770408 to CG), and (Grant No. 81900280 to XS).

Chang, C.-C., and Lin, C.-J. (2011). LIBSVM: a library for support vector machines. ACM Trans. on Intell. Syst. Technol. 2, 1-27. doi: 10.1145/1961189.196 1199

Chugh, S. S., Blackshear, J. L., Shen, W. K., Hammill, S. C., and Gersh, B. J. (2001). Epidemiology and natural history of atrial fibrillation: clinical implications. J. Am. Coll. Cardiol. 37, 371-378. doi: 10.1016/s0735-1097(00) 01107-4

Csardi, G., and Nepusz, T. (2006). The igraph software package for complex network research. Inter J. Complex Syst. 1695, 1-9.

Ellinor, P. T., Lunetta, K. L., Glazer, N. L., Pfeufer, A., Alonso, A., Chung, M. K., et al. (2010). Common variants in $\mathrm{KCNN} 3$ are associated with lone atrial fibrillation. Nat. Genet. 42, 240-244. doi: 10.1038/ng.537

Hoffmann, S., Paone, C., Sumer, S. A., Diebold, S., Weiss, B., Roeth, R., et al. (2019). Functional characterization of rare variants in the shox 2 gene identified in sinus node dysfunction and atrial fibrillation. Front. Genet. 10:648. doi: 10.3389/fgene.2019.00648

Kim, D., Langmead, B., and Salzberg, S. L. (2015). HISAT: a fast spliced aligner with low memory requirements. Nat. Methods 12, 357-360. doi: 10.1038/nmeth. 3317

Kissel, J. T., Dimberg, E. L., Emslie-Smith, A. M., Selcen, D., and Keegan, B. M. (2009). A 49-year-old man with contractures, weakness, and cardiac arrhythmia. Neurology 72, 2036-2043. doi: 10.1212/01.wnl.0000350522. 42635.09

Kursa, M., and Rudnicki, W. (2010). Feature selection with the boruta package. J. Statist. Softw. Artic. 36, 1-13. doi: 10.18637/jss.v036.i11 
Lin, H., Yin, X., Xie, Z., Lunetta, K. L., Lubitz, S. A., Larson, M. G., et al. (2017). Methylome-wide association study of atrial fibrillation in framingham heart study. Sci. Rep. 7:40377. doi: 10.1038/srep40377

Lip, G. Y. H., Lane, D. A., Buller, H., and Apostolakis, S. (2013). Development of a novel composite stroke and bleeding risk score in patients with atrial fibrillation: the AMADEUS study. Chest 144, 1839-1847. doi: 10.1378/chest.131635

Ma, B., Wilker, E. H., Willis-Owen, S. A., Byun, H. M., Wong, K. C., Motta, V., et al. (2014). Predicting DNA methylation level across human tissues. Nucleic Acids Res. 42, 3515-3528. doi: 10.1093/nar/gkt1380

Ma, J. F., Yang, F., Mahida, S. N., Zhao, L., Chen, X., Zhang, M. L., et al. (2016). TBX5 mutations contribute to early-onset atrial fibrillation in chinese and caucasians. Cardiovasc. Res. 109, 442-450. doi: 10.1093/cvr/cvw003

Milton, A. G., Aykanat, V. M., Hamilton-Bruce, M. A., Nezic, M., Jannes, J., and Koblar, S. A. (2011). Association of the phosphodiesterase 4D (PDE4D) gene and cardioembolic stroke in an Australian cohort. Int. J. Stroke 6, 480-486. doi: 10.1111/j.1747-4949.2011.00616.x

Molina, C. E., Leroy, J., Richter, W., Xie, M., Scheitrum, C., Lee, I. O., et al. (2012). Cyclic adenosine monophosphate phosphodiesterase type 4 protects against atrial arrhythmias. J. Am. Coll. Cardiol. 59, 2182-2190. doi: 10.1016/j.jacc.2012. 01.060

Nielsen, J. B., Thorolfsdottir, R. B., Fritsche, L. G., Zhou, W., Skov, M. W., Graham, S. E., et al. (2018). Biobank-driven genomic discovery yields new insight into atrial fibrillation biology. Nat. Genet. 50, 1234-1239. doi: 10.1038/s41588-0180171-3

Ogawa, H., Senoo, K., An, Y., Shantsila, A., Shantsila, E., Lane, D. A., et al. (2017). Clinical features and prognosis in patients with atrial fibrillation and prior stroke: comparing the fushimi and darlington AF registries. eBio Med. 18, 199-203. doi: 10.1016/j.ebiom.2017.03.022

Olesen, M. S., Nielsen, M. W., Haunso, S., and Svendsen, J. H. (2014). Atrial fibrillation: the role of common and rare genetic variants. Eur. J. Hum. Genet. 22, 297-306. doi: 10.1038/ejhg.2013.139

Pertea, M., Pertea, G. M., Antonescu, C. M., Chang, T.-C., Mendell, J. T., and Salzberg, S. L. (2015). StringTie enables improved reconstruction of a transcriptome from RNA-seq reads. Nat. Biotechnol. 33, 290-295. doi: 10.1038/ nbt. 3122

Sakata, K., Shimizu, M., Ino, H., Yamaguchi, M., Terai, H., Fujino, N., et al. (2005). High incidence of sudden cardiac death with conduction disturbances and atrial cardiomyopathy caused by a nonsense mutation in the STA gene. Circulation 111, 3352-3358. doi: 10.1161/CIRCULATIONAHA.104. 527184

Schnabel, R. B., Sullivan, L. M., Levy, D., Pencina, M. J., Massaro, J. M., D'Agostino, R. B., et al. (2009). Development of a risk score for atrial fibrillation (Framingham Heart Study): a community-based cohort study. Lancet 373, 739-745. doi: 10.1016/S0140-6736(09)60443-8

Shen, K., Tu, T., Yuan, Z., Yi, J., Zhou, Y., Liao, X., et al. (2017). DNA methylation dysregulations in valvular atrial fibrillation. Clin. Cardiol. 40, 686-691. doi: $10.1002 /$ clc. 22715
Sinner, M. F., Tucker, N. R., Lunetta, K. L., Ozaki, K., Smith, J. G., Trompet, S., et al. (2014). Integrating genetic, transcriptional, and functional analyses to identify 5 novel genes for atrial fibrillation. Circulation 130, 1225-1235. doi: 10.1161/CIRCULATIONAHA.114.009892

Szklarczyk, D., Gable, A. L., Lyon, D., Junge, A., Wyder, S., Huerta-Cepas, J., et al. (2018). STRING v11: protein-protein association networks with increased coverage, supporting functional discovery in genome-wide experimental datasets. Nucleic Acids Res. 47, D607-D613. doi: 10.1093/nar/gky1131

Tao, H., Shi, K. H., Yang, J. J., and Li, J. (2016). Epigenetic mechanisms in atrial fibrillation: new insights and future directions. Trends Cardiovasc. Med. 26, 306-318. doi: 10.1016/j.tcm.2015.08.006

Vicente-Steijn, R., Kelder, T. P., Tertoolen, L. G., Wisse, L. J., Pijnappels, D. A., Poelmann, R. E., et al. (2017). RHOA-ROCK signalling is necessary for lateralization and differentiation of the developing sinoatrial node. Cardiovasc. Res. 113, 1186-1197. doi: 10.1093/cvr/cvx104

Voukalis, C., Lip, G. Y., and Shantsila, E. (2016). Emerging Tools for Stroke Prevention in Atrial Fibrillation. eBio Med. 4, 26-39. doi: 10.1016/j.ebiom.2016. 01.017

Ye, W., Wang, J., Song, Y., Yu, D., Sun, C., Liu, C., et al. (2015). A common Shox2-Nkx2-5 antagonistic mechanism primes the pacemaker cell fate in the pulmonary vein myocardium and sinoatrial node. Development 142, 25212532. doi: 10.1242/dev.120220

Yeh, Y. H., Ehrlich, J. R., Qi, X., Hebert, T. E., Chartier, D., and Nattel, S. (2007). Adrenergic control of a constitutively active acetylcholine-regulated potassium current in canine atrial cardiomyocytes. Cardiovasc. Res. 74, 406-415. doi: 10.1016/j.cardiores.2007.01.020

Zhao, G., Zhou, J., Gao, J., Liu, Y., Gu, S., Zhang, X., et al. (2017). Genome-wide DNA methylation analysis in permanent atrial fibrillation. Mol. Med. Rep. 16, 5505-5514. doi: 10.3892/mmr.2017.7221

Zhao, J., Liu, T., and Li, G. (2014). Relationship between two arrhythmias: sinus node dysfunction and atrial fibrillation. Arch. Med. Res. 45, 351-355. doi: 10. 1016/j.arcmed.2014.04.005

Zhong, J., Agha, G., and Baccarelli, A. A. (2016). The role of DNA Methylation in cardiovascular risk and disease: methodological aspects, study design, and data analysis for epidemiological studies. Circ. Res. 118, 119-131. doi: 10.1161/ CIRCRESAHA.115.305206

Conflict of Interest: The authors declare that the research was conducted in the absence of any commercial or financial relationships that could be construed as a potential conflict of interest.

Copyright (c) 2020 Liu, Shi, Ding, Lv, Qian, Zhu, Guo and Zhang. This is an openaccess article distributed under the terms of the Creative Commons Attribution License (CC BY). The use, distribution or reproduction in other forums is permitted, provided the original author(s) and the copyright owner(s) are credited and that the original publication in this journal is cited, in accordance with accepted academic practice. No use, distribution or reproduction is permitted which does not comply with these terms. 